Document downloaded from:

http://hdl.handle.net/10251/160898

This paper must be cited as:

Calatayud, J.; Cortés, J.; Dorini, FA.; Jornet, M. (2020). On a stochastic logistic population model with time-varying carrying capacity. Computational and Applied Mathematics. 39(4):116. https://doi.org/10.1007/s40314-020-01343-z

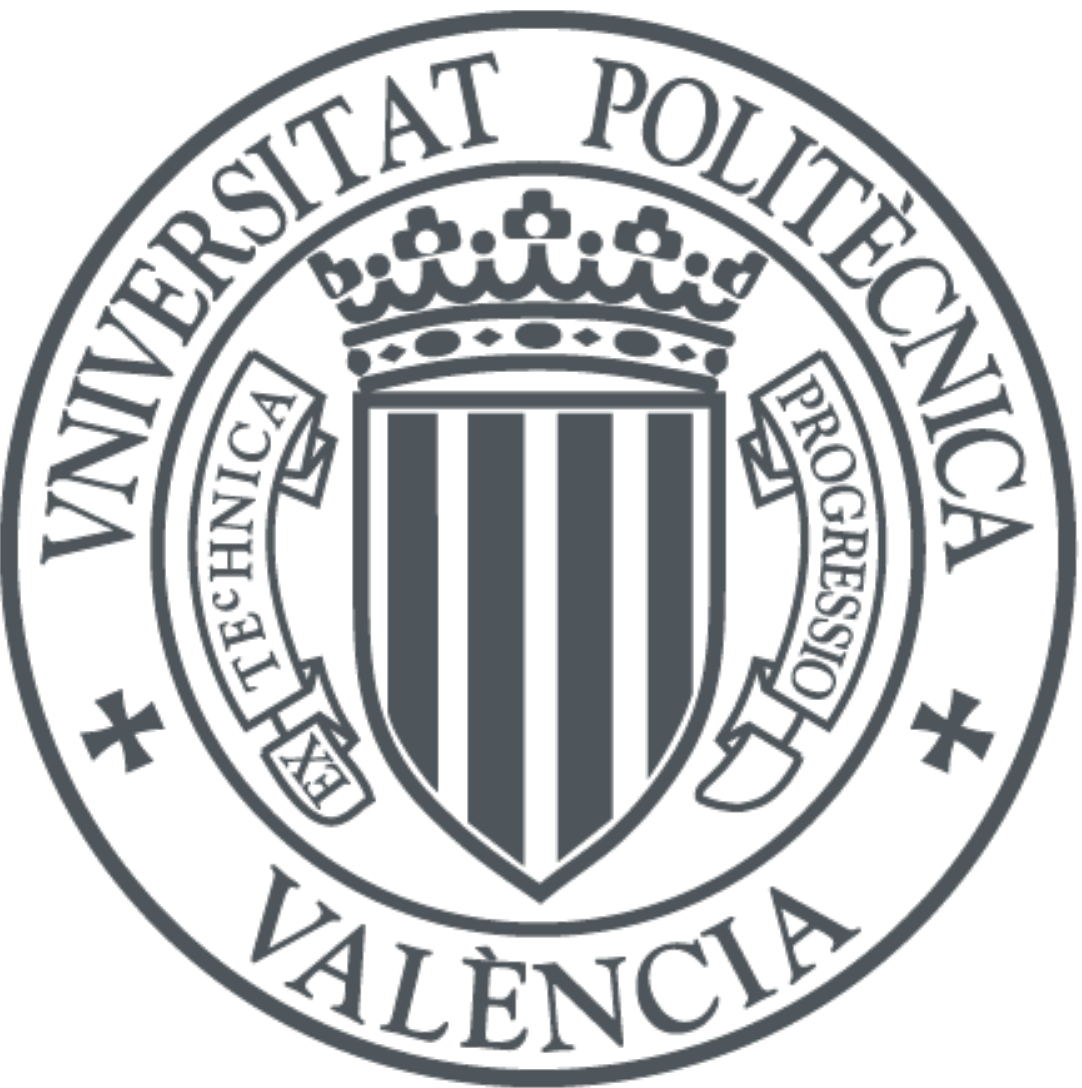

The final publication is available at

https://doi.org/10.1007/s40314-020-01343-z

Copyright Springer-Verlag

Additional Information 


\title{
ON A STOCHASTIC LOGISTIC POPULATION MODEL WITH TIME-VARYING CARRYING CAPACITY
}

\author{
J. CALATAYUD ${ }^{1}$, J.-C. CORTÉS ${ }^{1}$, F.A. DORINI ${ }^{2}$, M. JORNET ${ }^{1}$
}

\author{
${ }^{1}$ Instituto Universitario de Matemática Multidisciplinar, \\ Universitat Politècnica de València, \\ Camino de Vera s/n, 46022, Valencia, Spain \\ ${ }^{2}$ Department of Mathematics, \\ Federal University of Technology - Paraná \\ 80230-901, Curitiba, PR, Brazil
}

email: jucagre@doctor.upv.es; jccortes@imm.upv.es; fabio.dorini@gmail.com; marjorsa@doctor.upv.es

\begin{abstract}
In this paper, we deal with the logistic growth model with a timedependent carrying capacity that was proposed in the literature for the study of the total bacterial biomass during occlusion of healthy human skin. Accounting for data and model errors, randomness is incorporated into the equation by assuming that the input parameters are random variables. The uncertainty is quantified by approximations of the solution stochastic process via truncated series solution together with the random variable transformation method. Numerical examples illustrate the theoretical results.
\end{abstract}

Keywords: logistic growth model; time-dependent carrying capacity; random parameters; probability density function.

AMS Classification 2010: 34F05; 92D25; 92D40.

\section{INTRODUCTION}

Growth models such as the logistic equation are widely studied and applied in population and ecological modeling. Classically, the carrying capacity of the logistic equation model has been considered constant. However, some works started to consider it as a function of time, motivated by the principle that a changing environment may result in a significant change in the limiting capacity [1.

It is the case of the model proposed in [1, 2] for the study of total bacterial biomass during occlusion of healthy human skin. The model is presented by the non-autonomous logistic equation

$$
\left\{\begin{array}{l}
N^{\prime}(t)=a N(t)\left(1-\frac{N(t)}{K(t)}\right), \quad t>0, \\
N(0)=N_{0},
\end{array}\right.
$$


99

where $N_{0}>0$ is the initial condition and $a>0$ is the growth rate parameter, driven by the time-varying capacity, $K(t)$, that takes the form

$$
K(t)=K_{s}\left[1-\left(1-\frac{K_{0}}{K_{s}}\right) \mathrm{e}^{-c t}\right],
$$

where $K_{0}=K(0)$ is the initial limiting capacity, $K_{s}=\lim _{t \rightarrow+\infty} K(t)$ is the bacterial saturation (or equilibrium) level, and $c>0$ is the saturation constant. It is assumed $N_{0}<K_{0}<K_{s}$.

This model assumes that on the unoccluded skin the environment is relatively constant and the density of microbes is in equilibrium with its environment $\left(K_{0} \approx\right.$ $N_{0}$ ). After an occlusion is applied to the skin, the environment beneath it begins to change to one that is generally more favorable for microbial growth.

Equation (1.1) is a Bernoulli ordinary differential equation. After a classical change of variables, its solution can be presented as

$$
N(t)=\frac{\mathrm{e}^{a t} N_{0}}{1+a N_{0} \int_{0}^{t} \frac{\mathrm{e}^{a s}}{K(s)} \mathrm{d} s} .
$$

When $K(t)$ in $(1.2)$ is substituted into (1.3), we obtain the solution derived in [1]:

$$
N(t)=\frac{\mathrm{e}^{a t} N_{0}}{1+\frac{a N_{0}}{K_{s}} \int_{0}^{t} \frac{\mathrm{e}^{a s}}{1-b \mathrm{e}^{-c s}} \mathrm{~d} s},
$$

where $b=1-K_{0} / K_{s} \in(0,1)$.

To evaluate the integral in (1.4), the authors in [1] expanded part of the integrand as a convergent geometric series with ratio $b e^{-c s} \in(0,1)$,

$$
\frac{1}{1-b \mathrm{e}^{-c s}}=\sum_{n=0}^{\infty} b^{n} \mathrm{e}^{-n c s}
$$

so that

$$
\int_{0}^{t} \frac{\mathrm{e}^{a s}}{1-b \mathrm{e}^{-c s}} \mathrm{~d} s=\int_{0}^{t}\left(\sum_{n=0}^{\infty} b^{n} \mathrm{e}^{(a-n c) s}\right) \mathrm{d} s=\sum_{n=0}^{\infty} \frac{b^{n}}{a-n c}\left(\mathrm{e}^{(a-n c) t}-1\right) .
$$

Thus,

$$
N(t)=\frac{\mathrm{e}^{a t} N_{0}}{1+\frac{a N_{0}}{K_{s}} \sum_{n=0}^{\infty} \frac{b^{n}}{a-n c}\left(\mathrm{e}^{(a-n c) t}-1\right)} .
$$

In practice, the series in 1.5$)$ is truncated to a finite-term sum. Accurate approximations to the exact solution $N(t)$ are obtained for small orders of truncation of the series.

In the mathematical modeling of bacterial growth, the parameters are either measured directly or determined by curve fitting. These parameters may have large variability that depends on the experimental method and its inherent error, on differences in the actual population sample size used, as well as other factors that are difficult to account for. In view of this, randomness is incorporated into equation 
(1.1) by assuming that the input parameters $a, c, N_{0}, K_{0}$, and $K_{s}$ are random variables with known probability distributions. Therefore, the general solution $N(t)$ to (1.1), given by (1.5), becomes a random variable that evolves with time, that is, a stochastic process [3]. In this paper, we will assume that these random variables and stochastic process are defined in a complete probability space $(\Omega, \mathcal{F}, \mathbb{P})$, where $\Omega$ is the sample space consisting of outcomes $\omega \in \Omega, \mathcal{F}$ is the $\sigma$-algebra of events and $\mathbb{P}$ is the probability measure.

The aim of this work is to provide approximations of the (first) probability density function, $f_{N}(q ; t)$, of the solution stochastic process $N(t)$ in $[1.5)$, [3, Ch. 1]. By definition, the probability density function is a non-negative function characterized by $\mathbb{P}[N(t) \in \mathcal{B}]=\int_{\mathcal{B}} f_{N}(q ; t) \mathrm{d} q$ for any Borel set $\mathcal{B}$ in $\mathbb{R}$. A random variable or vector is said to be absolutely continuous when it has a probability density function.

The paper is organized as follows. In Section 2, an approximation of the first probability density function of the solution stochastic process to (1.1) is constructed. This approximation is based on the truncated series solution together with the random variable transformation method. Some results on the convergence of the aforementioned approximations of the probability density function of the solution are also presented. In Section 3, we determine a closed expression (via an integral) for the probability density function of the time-varying carrying capacity (1.2). Section 4 is addressed to show two illustrative examples where the proposed technique is successfully applied. Finally, in Section 5 our main conclusions are drawn.

\section{Approximation of the Density function of the SOLUtion PRoCess}

In this section, we assume that the random parameters of the model (1.1) have specific probability distributions, and then we compute approximations of the probability density function of its solution $N(t)$, for a fixed $t>0$, given by (1.5). For this, the series in (1.5) is truncated to a finite-term sum and then the random variable transformation method is employed to compute the density function.

Let $a, c, N_{0}, K_{0}$, and $K_{s}$ be absolutely continuous real random variables in $(\Omega, \mathcal{F}, \mathbb{P})$. Obviously, all of them depend on the sample parameter, for example $a=a(\omega), \omega \in \Omega$, but as usual this notation will be hidden hereinafter. We also assume that $a, c, N_{0}, K_{0}$, and $K_{s}$ are non-negative random variables and $N_{0}<K_{0}<K_{s}$.

The approximation of the first probability density function, $f_{N}(q ; t)$, of the stochastic process $N(t)$ given by (1.5), will be computed from the truncation, say $N^{p}(t)$, of $N(t)$,

$$
N^{p}(t)=\frac{\mathrm{e}^{a t} N_{0}}{1+\frac{a N_{0}}{K_{s}} \Lambda^{p}},
$$

where

$$
\Lambda^{p}=\Lambda^{p}\left(p, t ; a, c, K_{0}, K_{s}\right)=\sum_{n=0}^{p} \frac{b^{n}}{a-n c}\left(\mathrm{e}^{(a-n c) t}-1\right),
$$

$b=1-K_{0} / K_{s} \in(0,1)$, being $p$ a non-negative integer previously fixed. Truncation is required to keep the approximation to $f_{N}(q ; t)$ computationally feasible. 
95

96

97

103

To apply the random variable transformation method [4, Th. 2.1.5], [5, Th. 1], let us consider the mapping

$$
\left(a, c, N_{0}, K_{0}, K_{s}\right) \quad \mapsto \quad\left(X, Y, N^{p}, Z, W\right)=\left(a, c, \frac{\mathrm{e}^{a t} N_{0}}{1+\frac{a N_{0}}{K_{s}} \Lambda^{p}}, K_{0}, K_{s}\right),
$$

where the auxiliary random variables $X=a, Y=c, Z=K_{0}$, and $W=K_{s}$ have been conveniently chosen, and $N^{p}=N^{p}(t)$, for a fixed $t>0$.

It is not difficult to verify that the function defined by $(2.3)$ is invertible and its inverse is given by

$$
\left(X, Y, N^{p}, Z, W\right) \mapsto\left(a, c, N_{0}, K_{0}, K_{s}\right)=\left(X, Y, \frac{N^{p} W}{W \mathrm{e}^{X t}-X N^{p} \Lambda^{p}}, Z, W\right)
$$

where, according to 2.2 ,

$$
\Lambda^{p}=\Lambda^{p}(p, t ; X, Y, Z, W)=\sum_{n=0}^{p} \frac{b^{n}}{X-n Y}\left(\mathrm{e}^{(X-n Y) t}-1\right),
$$

$b=1-Z / W$.

From the random variable transformation method, the density function of $N^{p}(t)$, for a fixed $t>0$, can be presented as

$$
\begin{aligned}
& f_{N^{p}}\left(N^{p} ; t\right)=\int_{\mathcal{D}(X, Y, Z, W)} f_{\left(X, Y, N^{p}, Z, W\right)}\left(X, Y, N^{p}, Z, W\right) \mathrm{d} X \mathrm{~d} Y \mathrm{~d} Z \mathrm{~d} W= \\
& =\int_{\mathcal{D}\left(a, c, K_{0}, K_{s}\right)} f_{\left(a, c, N_{0}, K_{0}, K_{s}\right)}\left(a, c, N_{0}, K_{0}, K_{s}\right)\left|J\left(X, Y, N^{p}, Z, W\right)\right| \mathrm{d} a \mathrm{~d} c \mathrm{~d} K_{0} \mathrm{~d} K_{s},
\end{aligned}
$$

where $f_{\left(X, Y, N^{p}, Z, W\right)}$ is the joint density of the random variables $X, Y, N^{p}, Z$ and $W$; $f_{\left(a, c, N_{0}, K_{0}, K_{s}\right)}$ is the joint density of $a, c, N_{0}, K_{0}$, and $K_{s} ; \mathcal{D}$ denotes the support of the corresponding random vector; and $J\left(X, Y, N^{p}, Z, W\right)$ is the determinant Jacobian of the function given by $(2.4)$, that is,

$$
J\left(X, Y, N^{p}, Z, W\right)=\operatorname{det}\left(\frac{\partial\left(a, c, N_{0}, K_{0}, K_{s}\right)}{\partial\left(X, Y, N^{p}, Z, W\right)}\right)=\frac{\partial N_{0}}{\partial N^{p}}=\frac{W^{2} \mathrm{e}^{X t}}{\left(W \mathrm{e}^{X t}-X N^{p} \Lambda^{p}\right)^{2}}>0,
$$

where $\Lambda^{p}$ is given by (2.5).

Summarizing, the following result has been established.

Theorem 2.1. For a fixed $t>0$, the density function of $N^{p}(t), f_{N^{p}}\left(N^{p} ; t\right)$, given by (2.1), is

$$
f_{N^{p}}(q ; t)=\int_{\mathcal{D}\left(a, c, K_{0}, K_{s}\right)} f_{\left(a, c, N_{0}, K_{0}, K_{s}\right)}\left(a, c, \frac{q K_{s}}{K_{s} \mathrm{e}^{a t}-a q \Lambda^{p}}, K_{0}, K_{s}\right) \times
$$

$$
\times \frac{K_{s}^{2} \mathrm{e}^{a t}}{\left(K_{s} \mathrm{e}^{a t}-a q \Lambda^{p}\right)^{2}} \mathrm{~d} a \mathrm{~d} c \mathrm{~d} K_{0} \mathrm{~d} K_{s},
$$


where

$$
\Lambda^{p}=\sum_{n=0}^{p} \frac{b^{n}}{a-n c}\left(\mathrm{e}^{(a-n c) t}-1\right),
$$

$b=1-K_{0} / K_{s} \in(0,1)$, being $p$ a non-negative integer previously fixed.

It is important to observe that when some input random parameter is independent of the rest, then the joint density function in the integrand can be factorized as a product. For example, in the particular case that $a, c, N_{0}, K_{0}$, and $K_{s}$ are independent random variables, the integrand of (2.6) writes

$f_{\left(a, c, N_{0}, K_{0}, K_{s}\right)}\left(a, c, \frac{q K_{s}}{K_{s} \mathrm{e}^{a t}-a q \Lambda^{p}}, K_{0}, K_{s}\right)=f_{a}(a) f_{c}(c) f_{N_{0}}\left(\frac{q K_{s}}{K_{s} \mathrm{e}^{a t}-a q \Lambda^{p}}\right) f_{K_{0}}\left(K_{0}\right) f_{K_{s}}\left(K_{s}\right)$.

In general, we expect to have $\lim _{p \rightarrow \infty} f_{N^{p}}(q ; t)=f_{N}(q ; t)$ for all $q \in \mathbb{R}, t>0$. The following result provides general sufficient conditions so that this limit fulfills.

Theorem 2.2. Suppose that the random vector $\left(a, c, K_{0}, K_{s}\right)$ and the random variable $N_{0}$ are independent. Fix $t>0$. Assume that $\mathbb{E}\left[\mathrm{e}^{a t}\right]<\infty$ (i.e. the momentgenerating function of $a$ is finite at $t$ ). Suppose that the density function $f_{N_{0}}$ is continuous almost everywhere on $\mathbb{R}$ and satisfies $f_{N_{0}}(q) \leq C / q^{2}$ for almost every $q \in \mathbb{R} \backslash\{0\}$, where $C>0$ is a constant. Then $\lim _{p \rightarrow \infty} f_{N^{p}}(q ; t)=f_{N}(q ; t)$ for all $q \in \mathbb{R}$. Also $\lim _{p \rightarrow \infty} \int_{\mathbb{R}}\left|f_{N^{p}}(q ; t)-f_{N}(q ; t)\right| \mathrm{d} q=0$.

Proof. Because of the independence, the joint density function factorizes as $f_{\left(a, c, N_{0}, K_{0}, K_{s}\right)}\left(a, c, \frac{q K_{s}}{K_{s} \mathrm{e}^{a t}-a q \Lambda^{p}}, K_{0}, K_{s}\right)=f_{\left(a, c, K_{0}, K_{s}\right)}\left(a, c, K_{0}, K_{s}\right) f_{N_{0}}\left(\frac{q K_{s}}{K_{s} \mathrm{e}^{a t}-a q \Lambda^{p}}\right)$. Therefore, using the fact that the expectation is given by the integral with respect to the density function, we have

$$
f_{N^{p}}(q ; t)=\mathbb{E}\left[f_{N_{0}}\left(\frac{q K_{s}}{K_{s} \mathrm{e}^{a t}-a q \Lambda^{p}}\right) \frac{K_{s}^{2} \mathrm{e}^{a t}}{\left(K_{s} \mathrm{e}^{a t}-a q \Lambda^{p}\right)^{2}}\right] .
$$

We know that $\lim _{p \rightarrow \infty} \Lambda^{p}=\Lambda^{\infty}$ almost surely, where $\Lambda^{\infty}=\sum_{n=0}^{\infty} \frac{b^{n}}{a-n c}\left(\mathrm{e}^{(a-n c) t}-1\right)$. Since $f_{N_{0}}$ is continuous almost everywhere on $\mathbb{R}$, the continuous mapping theorem [6, p. 7, Th. 2.3] implies

$\lim _{p \rightarrow \infty} f_{N_{0}}\left(\frac{q K_{s}}{K_{s} \mathrm{e}^{a t}-a q \Lambda^{p}}\right) \frac{K_{s}^{2} \mathrm{e}^{a t}}{\left(K_{s} \mathrm{e}^{a t}-a q \Lambda^{p}\right)^{2}}=f_{N_{0}}\left(\frac{q K_{s}}{K_{s} \mathrm{e}^{a t}-a q \Lambda^{\infty}}\right) \frac{K_{s}^{2} \mathrm{e}^{a t}}{\left(K_{s} \mathrm{e}^{a t}-a q \Lambda^{\infty}\right)^{2}}$ almost surely. On the other hand, from the condition $f_{N_{0}}(q) \leq C / q^{2}$, we bound

$$
f_{N_{0}}\left(\frac{q K_{s}}{K_{s} \mathrm{e}^{a t}-a q \Lambda^{p}}\right) \frac{K_{s}^{2} \mathrm{e}^{a t}}{\left(K_{s} \mathrm{e}^{a t}-a q \Lambda^{p}\right)^{2}} \leq \frac{C \mathrm{e}^{a t}}{q^{2}} \in \mathrm{L}^{1}(\Omega ; \mathrm{d} \mathbb{P}),
$$

for $q \in \mathbb{R} \backslash\{0\}$. By the dominated convergence theorem [7, result 11.32, p. 321], we can interchange the limit with respect to $p$ and the expectation:

$$
\lim _{p \rightarrow \infty} f_{N^{p}}(q ; t)=\mathbb{E}\left[f_{N_{0}}\left(\frac{q K_{s}}{K_{s} \mathrm{e}^{a t}-a q \Lambda^{\infty}}\right) \frac{K_{s}^{2} \mathrm{e}^{a t}}{\left(K_{s} \mathrm{e}^{a t}-a q \Lambda^{\infty}\right)^{2}}\right]=f_{N}(q ; t) .
$$

Finally, convergence in $\mathrm{L}^{1}(\mathbb{R} ; \mathrm{d} q)$ follows from Scheffé's lemma [8, p. 55], [9]. This lemma states that if a general sequence of integrable functions converges almost everywhere to another integrable function, then convergence in $L^{1}(\mathbb{R})$ is equivalent 
to convergence of the $L^{1}(\mathbb{R})$ norms (the $L^{1}(\mathbb{R})$ norm of all density functions being equal to 1$)$.

The following result provides sufficient conditions to assess the behavior of $f_{N}(q ; t)$ for large values of $t$.

Theorem 2.3. Suppose that the random vector $\left(a, c, K_{0}, K_{s}\right)$ and the random variable $N_{0}$ are independent. Given the probability density function $f_{N}(q ; t)$ of $N(t)$, (2.7), if the density function $f_{N_{0}}$ is continuous almost everywhere on $\mathbb{R}$, and we have $\lim \sup _{q \rightarrow 0^{+}} f_{N_{0}}(q)<\infty$ for some representation of $f_{N_{0}}$, then $\lim _{t \rightarrow \infty} f_{N}(q ; t)=$ $f_{K_{s}}(q)$ for all $q \in \mathbb{R}$.

Proof. Given the governing equation (1.1), we may assume that $K_{s}=1$, by scaling out by $K_{s}$.

From representation (1.5), assuming $K_{s}=1$, we have

$$
N(t)=\frac{\mathrm{e}^{a t} N_{0}}{1+a N_{0} \Lambda^{\infty}},
$$

where $\Lambda^{\infty}=\Lambda^{\infty}\left(t ; K_{s}\right)=\sum_{n=0}^{\infty} \frac{b^{n}}{a-n c}\left(\mathrm{e}^{(a-n c) t}-1\right)$ (we make the dependence on $t$ and $K_{s}$ explicit). Simple calculations provide $N(t)=N_{0}\left(\mathrm{e}^{a t}-a \Lambda^{\infty} N(t)\right)$. We know that $N(t) \stackrel{t \rightarrow \infty}{\longrightarrow} K_{s}=1$. Then one verifies that $N_{0}\left(\mathrm{e}^{a t}-a \Lambda^{\infty}\right) \stackrel{t \rightarrow \infty}{\longrightarrow} 1$, that is,

$$
\mathrm{e}^{a t}-a \Lambda^{\infty} \stackrel{t \rightarrow \infty}{\longrightarrow} 1 / N_{0}
$$

almost surely.

Moreover, since $N(t)=\left(\mathrm{e}^{a t} N_{0}\right) /\left(1+a N_{0} \Lambda^{\infty}\right) \stackrel{t \rightarrow \infty}{\longrightarrow} 1$ and $\mathrm{e}^{a t} \stackrel{t \rightarrow \infty}{\longrightarrow} \infty$, it follows that $\left(\mathrm{e}^{a t} N_{0}\right) /\left(a N_{0} \Lambda^{\infty}\right) \stackrel{t \rightarrow \infty}{\longrightarrow} 1$, that is,

$$
\mathrm{e}^{a t} /\left(a \Lambda^{\infty}\right) \stackrel{t \rightarrow \infty}{\longrightarrow} 1
$$

almost surely.

If $q=1$, then

$f_{N_{0}}\left(\frac{q K_{s}}{K_{s} \mathrm{e}^{a t}-a q \Lambda^{\infty}}\right) \frac{K_{s}^{2} \mathrm{e}^{a t}}{\left(K_{s} \mathrm{e}^{a t}-a q \Lambda^{\infty}\right)^{2}}=f_{N_{0}}\left(\frac{1}{\mathrm{e}^{a t}-a \Lambda^{\infty}}\right) \frac{\mathrm{e}^{a t}}{\left(\mathrm{e}^{a t}-a \Lambda^{\infty}\right)^{2}} \stackrel{t \rightarrow \infty}{\longrightarrow} \infty$

almost surely, because $1 /\left(\mathrm{e}^{a t}-a \Lambda^{\infty}\right) \stackrel{t \rightarrow \infty}{\longrightarrow} N_{0}, f_{N_{0}}$ is continuous almost everywhere on $\mathbb{R}, f_{N_{0}}\left(N_{0}\right)>0, \mathrm{e}^{a t} \stackrel{t \rightarrow \infty}{\longrightarrow} \infty$, and $1 /\left(\mathrm{e}^{a t}-a \Lambda^{\infty}\right)^{2} \stackrel{t \rightarrow \infty}{\longrightarrow} N_{0}^{2}>0$.

When applying expectation, by Fatou's lemma [7, result 11.31, pp. 320-321] we have $\lim _{t \rightarrow \infty} f_{N}(q=1 ; t)=\infty$.

In the case $q \neq 1$, from $\mathrm{e}^{a t} /\left(a \Lambda^{\infty}\right) \stackrel{t \rightarrow \infty}{\longrightarrow} 1$ and $\mathrm{e}^{a t} \stackrel{t \rightarrow \infty}{\longrightarrow} \infty$, we arrive at

$$
\mathrm{e}^{a t}-a q \Lambda^{\infty}=\mathrm{e}^{a t}\left(1-q \frac{a \Lambda^{\infty}}{\mathrm{e}^{a t}}\right) \stackrel{t \rightarrow \infty}{\longrightarrow} \infty
$$

and $\mathrm{e}^{a t} /\left(\mathrm{e}^{a t}-a q \Lambda^{\infty}\right)^{2}=1 /\left(\mathrm{e}^{a t}\left[1-q a \Lambda^{\infty} / \mathrm{e}^{a t}\right]^{2}\right) \stackrel{t \rightarrow \infty}{\longrightarrow} 0$ almost surely.

Since $\lim \sup _{q \rightarrow 0^{+}} f_{N_{0}}(q)<\infty$ by hypothesis, from $q /\left(\mathrm{e}^{a t}-a q \Lambda^{\infty}\right) \stackrel{t \rightarrow \infty}{\longrightarrow} 0$ it follows that $\lim \sup _{t \rightarrow \infty} f_{N_{0}}\left(q /\left(\mathrm{e}^{a t}-a q \Lambda^{\infty}\right)\right)<\infty$ almost surely. 
Thus, we conclude that

$f_{N_{0}}\left(\frac{q K_{s}}{K_{s} \mathrm{e}^{a t}-a q \Lambda^{\infty}}\right) \frac{K_{s}^{2} \mathrm{e}^{a t}}{\left(K_{s} \mathrm{e}^{a t}-a q \Lambda^{\infty}\right)^{2}}=f_{N_{0}}\left(\frac{q}{\mathrm{e}^{a t}-a q \Lambda^{\infty}}\right) \frac{\mathrm{e}^{a t}}{\left(\mathrm{e}^{a t}-a q \Lambda^{\infty}\right)^{2}} \stackrel{t \rightarrow \infty}{\longrightarrow} 0$ almost surely. When applying expectation, by Fatou's lemma (lim sup version) we derive that $\lim _{t \rightarrow \infty} f_{N}(q \neq 1 ; t)=0$.

Since $\int_{\mathbb{R}} f_{N}(q ; t) \mathrm{d} q=1$ for all $t>0$, we have a heuristic representation of the Dirac delta function centered at 1 when $t \rightarrow \infty$, which is the probability density function of $K_{s}=1$.

\section{Approximation of the Density FUnCTion of THE TIME-VARYing CARRYING CAPACITY}

In the previous section, we constructed approximations of the probability density function of the solution and we gave sufficient conditions to guarantee that such approximations converge. Now we address the computation of the density function of the time-dependent carrying capacity, $f_{K}(q ; t)$, given by 1.2$)$. As it shall be seen, the random variable transformation method permits determining an integral expression for $f_{K}(q ; t)$. The results will be applied later in Examples 4.1 and 4.2 .

To obtain $f_{K}(q ; t)$, let us apply the random variable transformation method with the following mapping:

$$
\left(c, K_{0}, K_{s}\right) \quad \mapsto \quad(X, K, Y)=\left(c, K_{s}\left[1-\left(1-\frac{K_{0}}{K_{s}}\right) \mathrm{e}^{-c t}\right], K_{s}\right) .
$$

Its inverse is given by the map

$$
(X, K, Y) \mapsto\left(c, K_{0}, K_{s}\right)=\left(X, K \mathrm{e}^{X t}-Y\left(\mathrm{e}^{X t}-1\right), Y\right),
$$

with determinant Jacobian

$$
J(X, K, Y)=\operatorname{det}\left(\frac{\partial\left(c, K_{0}, K_{s}\right)}{\partial(X, K, Y)}\right)=\frac{\partial K_{0}}{\partial K}=\mathrm{e}^{X t}>0 .
$$

This yields

$$
f_{K}(q ; t)=\int_{\mathcal{D}\left(c, K_{s}\right)} f_{\left(c, K_{0}, K_{s}\right)}\left(c, q \mathrm{e}^{c t}-K_{s}\left(\mathrm{e}^{c t}-1\right), K_{s}\right) \mathrm{e}^{c t} \mathrm{~d} c \mathrm{~d} K_{s} .
$$

Similarly, we can choose the following mapping:

$$
\left(c, K_{0}, K_{s}\right) \mapsto(K, X, Y)=\left(K_{s}\left[1-\left(1-\frac{K_{0}}{K_{s}}\right) \mathrm{e}^{-c t}\right], K_{0}, K_{s}\right) .
$$

Its inverse is given by

$$
(K, X, Y) \mapsto \quad\left(c, K_{0}, K_{s}\right)=\left(\frac{1}{t} \ln \left(\frac{Y-X}{Y-K}\right), X, Y\right),
$$

with determinant Jacobian

$$
J(K, X, Y)=\operatorname{det}\left(\frac{\partial\left(c, K_{0}, K_{s}\right)}{\partial(K, X, Y)}\right)=\frac{\partial c}{\partial K}=\frac{1}{t(Y-K)}>0,
$$


since $Y=K_{s}>K=K(t)$ for all $t>0$. This allows us to present $f_{K}(q ; t)$ as

$$
f_{K}(q ; t)=\int_{\mathcal{D}\left(K_{0}, K_{s}\right)} f_{\left(c, K_{0}, K_{s}\right)}\left(\frac{1}{t} \ln \left(\frac{K_{s}-K_{0}}{K_{s}-q}\right), K_{0}, K_{s}\right) \frac{1}{t\left(K_{s}-q\right)} \mathrm{d} K_{0} \mathrm{~d} K_{s} .
$$

Just as a remark, if we consider $c$ and $K_{0}$ as independent random variables and $K_{s}=1$ (with density function considered in terms of the Dirac delta function, as before), then we obtain from $(3.1)$ and $(3.2)$

$$
\begin{aligned}
f_{K}(q ; t) & =\int_{\mathcal{D}(c)} f_{\left(c, K_{0}\right)}\left(c,(q-1) \mathrm{e}^{c t}+1\right) \mathrm{e}^{c t} \mathrm{~d} c \\
& =\int_{\mathcal{D}(c)} f_{c}(c) f_{K_{0}}\left((q-1) \mathrm{e}^{c t}+1\right) \mathrm{e}^{c t} \mathrm{~d} c \\
& =\mathbb{E}\left[f_{K_{0}}\left((q-1) \mathrm{e}^{c t}+1\right) \mathrm{e}^{c t}\right]
\end{aligned}
$$

and

$$
\begin{aligned}
f_{K}(q ; t) & =\int_{\mathcal{D}\left(K_{0}\right)} f_{\left(c, K_{0}\right)}\left(\frac{1}{t} \ln \left(\frac{1-K_{0}}{1-q}\right), K_{0}\right) \frac{1}{t(1-q)} \mathrm{d} K_{0} \\
& =\int_{\mathcal{D}\left(K_{0}\right)} f_{K_{0}}\left(K_{0}\right) f_{c}\left(\frac{1}{t} \ln \left(\frac{1-K_{0}}{1-q}\right)\right) \frac{1}{t(1-q)} \mathrm{d} K_{0} \\
& =\mathbb{E}\left[f_{c}\left(\frac{1}{t} \ln \left(\frac{1-K_{0}}{1-q}\right)\right) \frac{1}{t(1-q)}\right],
\end{aligned}
$$

respectively.

\section{NUMERICAL EXAMPLES}

This section is addressed to show two examples where the previous results are illustrated.

Example 4.1. Let us suppose that the random variable $a$ follows a uniform distribution on $[0.13,0.17], c$ has an exponential distribution with rate parameter $10, N_{0}$ has a uniform distribution on [0.19,0.21], $K_{0}$ is uniform on [0.26, 0.34], and $K_{s}=1$ (it represents the maximum proportion; its density function may be considered in terms of the Dirac delta function). Moreover, all the involved random variables are assumed to be independent.

We point out that the uniform distribution corresponds to the maximum entropy distribution when only prior information about the bounded support is known, while the exponential distribution is the maximum entropy distribution for a positive random quantity with known mean value [10, 11]. In modeling, the support and the mean value of an input random parameter may be inferred from its physical interpretation, experimental measurements or curve fittings.

Since $a$ is bounded, we have $\mathbb{E}\left[\mathrm{e}^{a t}\right]<\infty$ for all $t>0$. On the other hand, $f_{N_{0}}$ is continuous except at the points 0.19 and 0.21 (hence continuous almost everywhere on $\mathbb{R}$ ), and satisfies $f_{N_{0}}(q) \leq C / q^{2}$ for some $C>0$ because it has bounded support. Thus, the conditions of Theorem 2.2 hold, therefore $\lim _{p \rightarrow \infty} f_{N^{p}}(q ; t)=f_{N}(q ; t)$ for all $q \in \mathbb{R}$, and $\lim _{p \rightarrow \infty} \int_{\mathbb{R}}\left|f_{N^{p}}(q ; t)-f_{N}(q ; t)\right| \mathrm{d} q=0$, for any $t>0$.

To illustrate our results, in Figure 1 we present approximations of $f_{N^{p}}(q ; 5)$ and $f_{N^{p}}(q ; 10)$ given in 2.6$)$ for several values of $p$. They were computed by using the 
crude Monte Carlo method with realizations from $a \sim \operatorname{Uniform}(0.13,0.17), c \sim$ Exponential(10), and $K_{0} \sim \operatorname{Uniform}(0.26,0.34)$, to estimate the expectation

$$
f_{N^{p}}(q ; t)=\mathbb{E}\left[f_{N_{0}}\left(\frac{q}{\mathrm{e}^{a t}-a q \Lambda^{p}}\right) \frac{\mathrm{e}^{a t}}{\left(\mathrm{e}^{a t}-a q \Lambda^{p}\right)^{2}}\right]
$$

parametrically, with $\Lambda^{p}$ given in $(2.2)$. As the integrand in (2.6) has jump discontinuities in $f_{N_{0}}$ (the convergence becomes slow and we would have to deal with numerical instabilities), the Monte Carlo method has been utilized instead of computing the integral via quadrature techniques.
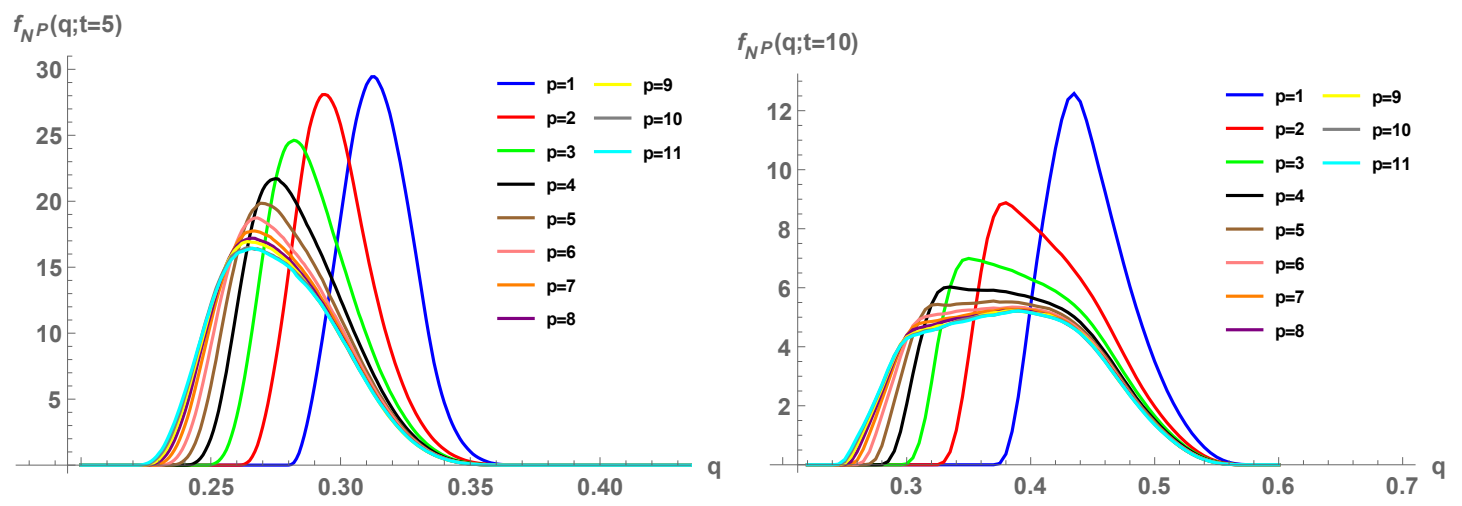

FiguRE 1. Approximations of $f_{N}(q ; 5)$ and $f_{N}(q ; 10)$ for several values of $p$.

We also compare the densities $f_{N^{11}}(q ; 5)$ and $f_{N^{11}}(q ; 10)$ above with those ones obtained by employing a kernel density estimation method (SmoothKernelDistribution function from the Mathematica software [12], with Gaussian kernel and Silverman's selection of the bandwidth) with 2000000 realizations of $a, c, N_{0}$, and $K_{0}$. It is observed full agreement, see Figure 2 .
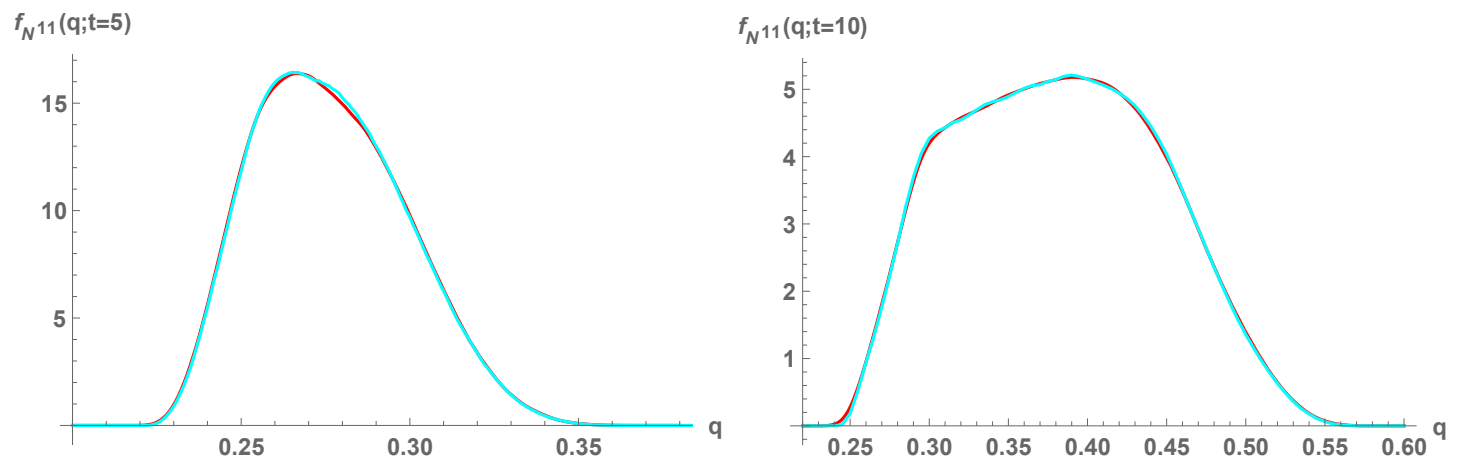

FiguRE 2. Approximations of $f_{N}(q ; 5)$ and $f_{N}(q ; 10)$. The cyan lines were computed using a kernel density estimation method, and the red lines are the densities $f_{N^{11}}(q ; 5)$ and $f_{N^{11}}(q ; 10)$ previously presented in Figure 1.

Figure 3 illustrates approximations of the density function of $K(t), f_{K}(q ; t)$, for several values of $t$. The red line represents an approximation of $f_{K}(q ; t)$ by computing 
the expectation in (3.4) using the crude Monte Carlo method with realizations of $K_{0}$. The blue line, calculated only to compare results, represents an approximation of $f_{K}(q ; t)$ using a kernel density estimation method (SmoothKernelDistribution function from the Mathematica software) with 1000000 realizations of $c$ and $K_{0}$. Notice that, in contrast to kernel density estimation (non-parametric nature), our parametric method is able to capture the density features (in this case non-differentiability points).
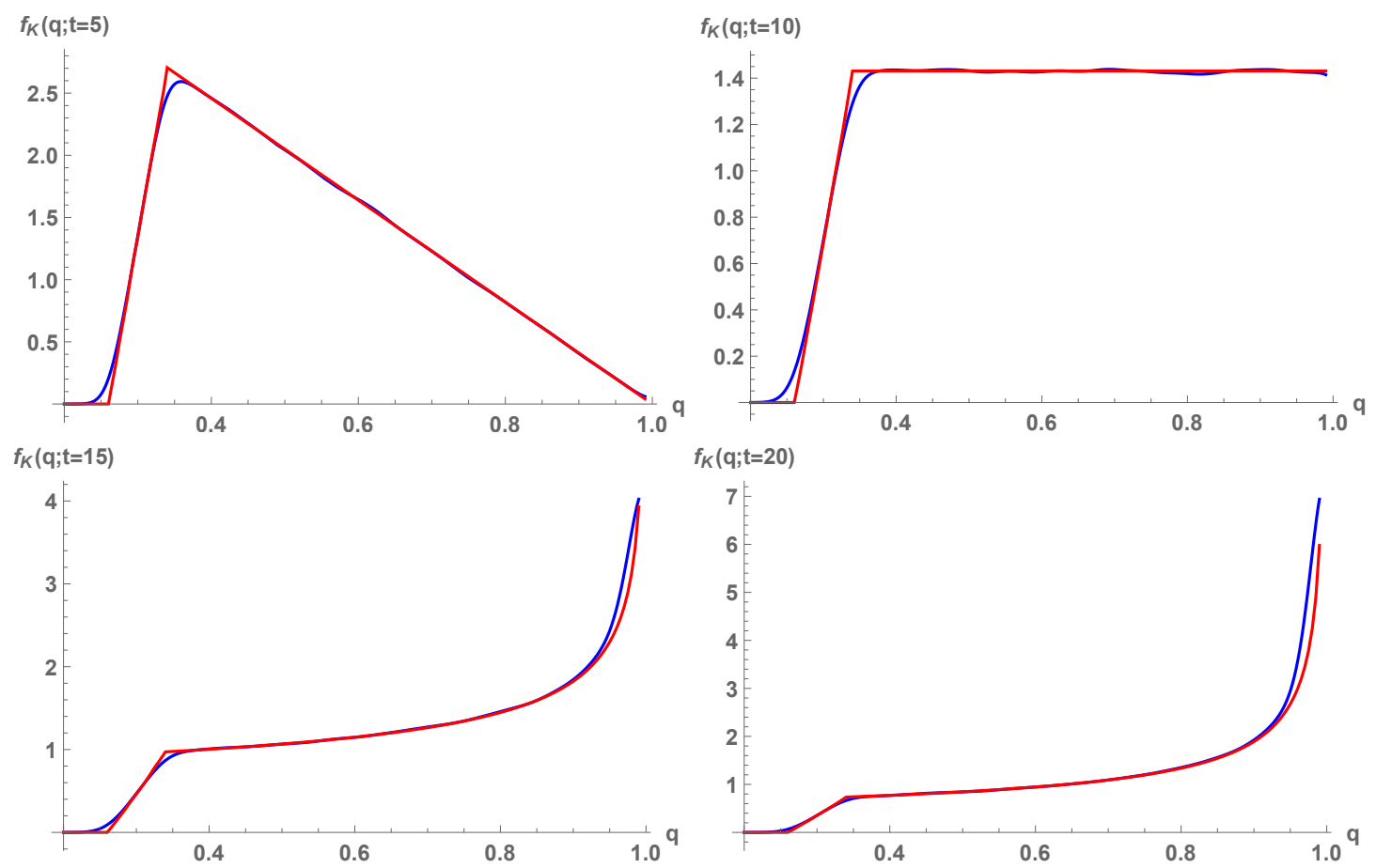

$f_{K}(q ; t=20)$

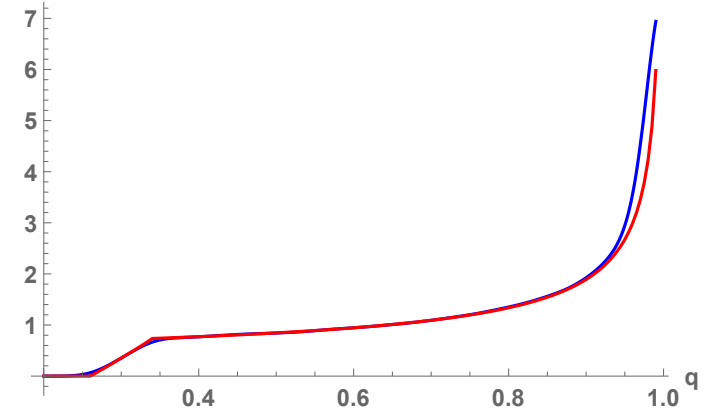

FiguRE 3 . Estimations of $f_{K}(q ; t)$ for several values of $t$. The red line represents the approximation by computing the expectation in (3.4) using the Monte Carlo method; the blue line represents the approximation of $f_{K}(q ; t)$ using a kernel density estimation method.

To emphasize the relevance of the variability of the parameters, we compare the expectation of $N(t)$ and $K(t), \mathbb{E}[N(t)]$ and $\mathbb{E}[K(t)]$, with the solution of the simplified version of (1.1) and (1.2), respectively, where the random parameters are replaced by their respective means, $\mathbb{E}[a]=0.15, \mathbb{E}[c]=0.1, \mathbb{E}\left[N_{0}\right]=0.2$, and $\mathbb{E}\left[K_{0}\right]=0.30$. Observe that $\mathbb{E}[N(t)]$ takes much more time than the solution of the simplified version of (1.1) to approach $K_{s}=1$. Figure 4 illustrates the two approaches: the fat line refers to the simplified version of $K(t)$; the red one refers to $\mathbb{E}[K(t)]$ computed using the crude Monte Carlo method; the dots correspond to the numerical solution of the simplified version of $N(t)$ employing the classical Runge-Kutta scheme; the green solid thin line represents the approximated solution, given by $(2.1)-(2.2)$ with $p=11$, of the simplified version of $N(t)$; the blue line refers to $\mathbb{E}[N(t)]$ computed using the crude Monte Carlo method. 


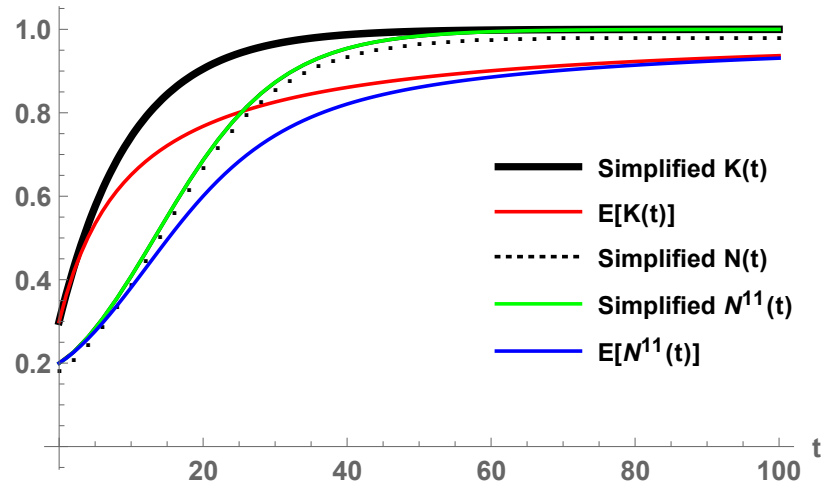

FiguRE 4. Simplified version of $K(t)$ (fat line); $\mathbb{E}[K(t)]$ (red line) computed using the Monte Carlo method; numerical solution of the simplified version of $N(t)$ (dots); approximated solution of the simplified version of $N^{11}(t)$ in 2.1)-2.2) (green line); $\mathbb{E}[N(t)]$ computed using the crude Monte Carlo method (blue line); $t \in[0,100]$.

Example 4.2. Now, let us assume that all the involved random variables, $a, c$, $N_{0}, K_{0}$, and $K_{s}$ are independent, $K_{s}=1$ (the maximum proportion, as before), and suppose that their mean values are known, that is, $\mathbb{E}[a]=0.15, \mathbb{E}[c]=0.10$, $\mathbb{E}\left[N_{0}\right]=0.20$, and $\mathbb{E}\left[K_{0}\right]=0.30$.

According to the maximum entropy principle [10, 11], the random parameters $a>0$ and $c>0$ follow an exponential distribution with rate parameters $1 / 0.15$ and $1 / 0.10$, respectively. These distributions maximize the ignorance on the random behavior of $a$ and $c$, while not violating the restrictions on their supports and mean values. On the other hand, since $N_{0} \in(0,1)$ and its mean value is known (and is less than 0.5), it follows that its maximum entropy distribution is the truncated exponential distribution with (approximated) rate parameter 4.80101, the unique solution to the nonlinear equation $1 /\left(1-\mathrm{e}^{x}\right)+1 / x=\mathbb{E}\left[N_{0}\right]=0.20$ [10, 11]. Similarly, $K_{0} \in(0,1)$ has a truncated exponential distribution with rate parameter 2.6721.

The moment-generating function of $a$ is given by $\mathbb{E}\left[\mathrm{e}^{a t}\right]=1 /(1-0.15 t)<+\infty$, for $t \in(0,1 / 0.15) \simeq(0,6.67)$. On the other hand, $f_{N_{0}}$ is continuous except at the points 0 and 1 (hence continuous almost everywhere on $\mathbb{R}$ ), and satisfies $f_{N_{0}}(q) \leq C / q^{2}$ for some $C>0$ because it has bounded support. Thus, the conditions of Theorem 2.2 hold for all $t \in(0,1 / 0.15)$. Therefore, $\lim _{p \rightarrow \infty} f_{N^{p}}(q ; t)=f_{N}(q ; t)$ for all $q \in \mathbb{R}$, and $\lim _{p \rightarrow \infty} \int_{\mathbb{R}}\left|f_{N^{p}}(q ; t)-f_{N}(q ; t)\right| \mathrm{d} q=0$, for any $t \in(0,1 / 0.15)$.

Figure 5 (left) illustrates our result for $t=5$. Although the hypothesis of Theorem 2.2 guarantees convergence only for $t \in(0,1 / 0.15)$, Figure 5 (right) indicates that the conditions on Theorem 2.2 could possibly be weakened. The plots in Figure 5 were computed by using the Monte Carlo method with realizations

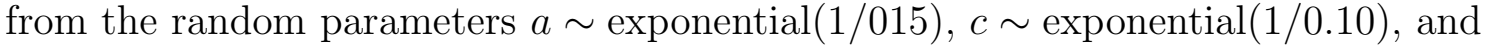
$K_{0} \sim$ truncated exponential $(2.6721)$ on $(0,1)$, to estimate the expectation in (4.1). Again, as the integrand in (2.6) has jump discontinuities in $f_{N_{0}}$ the Monte Carlo method has been utilized instead of computing the integral via quadrature techniques. 

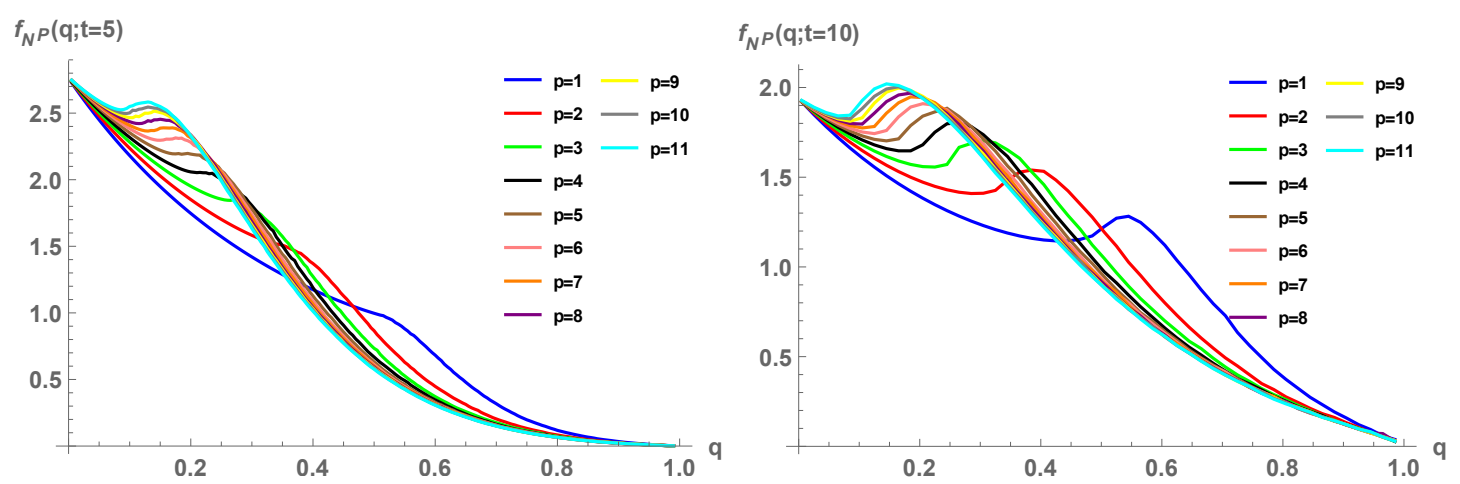

FiguRE 5. Approximations of $f_{N}(q ; 5)$ and $f_{N}(q ; 10)$ for several values of $p$.

Figure 6 illustrates the comparison of densities $f_{N^{11}}(q ; 5)$ and $f_{N^{11}}(q ; 10)$ above with those ones obtained by employing a kernel density estimation method. It is observed full agreement.
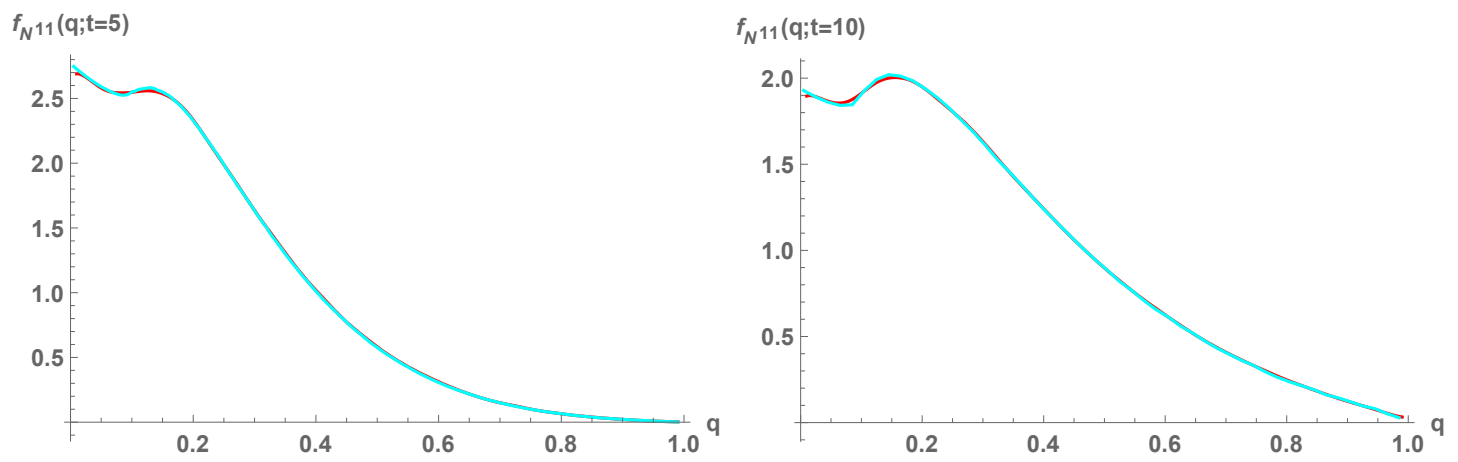

FiguRE 6 . Approximations of $f_{N}(q ; 5)$ and $f_{N}(q ; 10)$. The cyan lines were computed using a kernel density estimation method, and the red lines are the densities $f_{N^{11}}(q ; 5)$ and $f_{N^{11}}(q ; 10)$ previously presented in Figure 5 .

Figure 7 illustrates approximations of the density function of $K(t), f_{K}(q ; t)$, for several values of $t$.

As in Example 4.1, to emphasize the relevance of the variability of the parameters, we compare the expectation of $N(t)$ and $K(t)$ with the solution to the simplified version of (1.1) and (1.2), respectively. As before, $\mathbb{E}[N(t)]$ takes much more time than the solution to the simplified version of (1.1) to approach $K_{s}=1$. Figure 8 illustrates the two approaches.

\section{Conclusions}

In this paper we have extended, to the random setting, a non-autonomous logistic model whose carrying capacity is variable, to better describe changes in the environment. The original deterministic model depends on five parameters, which define the initial condition, the intrinsic growth and the variable carrying capacity, 

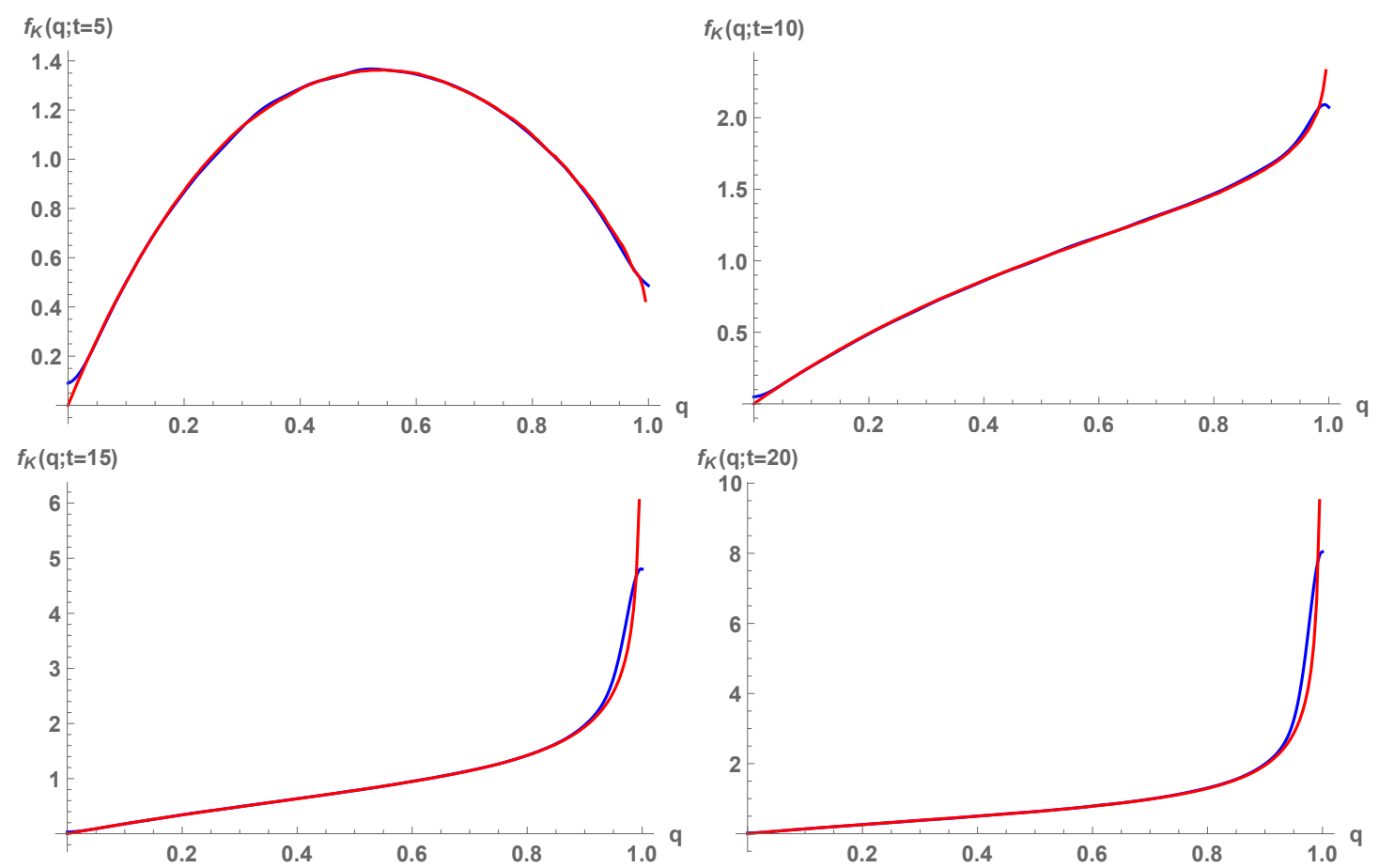

FiguRE 7. Estimations of $f_{K}(q ; t)$ for several values of $t$. The red line represents the approximation by computing the expectation in (3.4) using the Monte Carlo method; the blue line represents the approximation of $f_{K}(q ; t)$ using a kernel density estimation method.

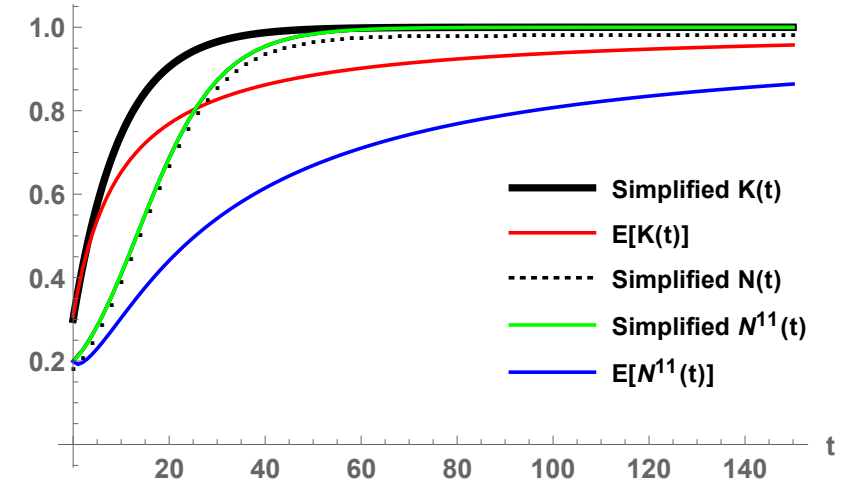

Figure 8. Simplified version of $K(t)$ (fat line); $\mathbb{E}[K(t)]$ (red line) computed using the Monte Carlo method; numerical solution of the simplified version of $N(t)$ (dots); approximated solution of the simplified version of $N^{11}(t)$ in 2.1$)-(2.2)$ (green line); $\mathbb{E}[N(t)]$ computed using the Monte Carlo method (blue line); $t \in[0,150]$.

and whose nature is clearly stochastic. Then, we have randomized all these model parameters and we have formulated its stochastic counterpart by assuming that these parameters are random variables instead of deterministic. By assuming mild 
differential equation via the computation of the probability density functions of the solution and of the carrying capacity, which are stochastic processes. The numerical examples confirm that our analysis extends consistently its deterministic counterpart. Therefore, our study provides a reliable approach to treat the aforementioned non-autonomous logistic model, which may result useful to consider uncertainties often met in dealing with ecological models. Although in our analysis we have assumed a particular functional form for the carrying-capacity, which has been applied in previous contributions by other authors, we do think that the approach may be successfully extended to other mathematical expressions and may open new avenues in the stochastic analysis of non-autonomous logistic-type models that have variable carrying capacity in their formulation.

\section{ACKNOWLEDGEMENTS}

This work has been supported by the Spanish Ministerio de Economía, Industria y Competitividad (MINECO), the Agencia Estatal de Investigación (AEI) and Fondo Europeo de Desarrollo Regional (FEDER UE) grant MTM2017-89664-P.

\section{Conflict of Interest Statement}

The authors declare that there is no conflict of interests regarding the publication of this article.

\section{REFERENCES}

[1] H.M. Safuan, Z. Jovanoski, I.N. Towers, and H.S. Sidhu. Exact solution of a non-autonomous logistic population model. Ecological Modelling, 251, 99-102 (2013).

[2] H. Safuan, I.N. Towers, Z. Jovanoski, and H.S. Sidhu. A simple model for the total microbial biomass under occlusion of healthy human skin. In MODSIM2011, 19th International Congress on Modelling and Simulation. Modelling and Simulation Society of Australia and New Zealand, pages 733-739 (2011).

[3] T. Neckel and F. Rupp. Random Differential Equations in Scientific Computing. Walter de Gruyter, 2013.

[4] G. Casella and R.L. Berger. Statistical Inference. Duxbury Pacific Grove, CA, 2 edition, 2002.

[5] M.C. Casabán, J.C. Cortés, A. Navarro-Quiles, J.V. Romero, M.D. Roselló, and M.D. Villanueva. A comprehensive probabilistic solution of random SIS-type epidemiological models using the random variable transformation technique. Communications in Nonlinear Science and Numerical Simulation, 32, 199-210 (2016).

[6] A.W. van der Vaart. Asymptotic Statistics. Cambridge University Press, ISBN: 9780521784504, 1998.

[7] W. Rudin. Principles of Mathematical Analysis. International Series in Pure \& Applied Mathematics, 3 edition, ISBN: 9780070542358, 1976.

[8] H. Scheffé. A useful convergence theorem for probability distributions. Ann. Math. Stat. 18(3), 434-438 (1947).

[9] L. Tenorio. An Introduction to Data Analysis and Uncertainty Quantification for Inverse Problems. Vol. 3, SIAM, 2017.

[10] F.A. Dorini and R. Sampaio. Some results on the random wear coefficient of the Archard model. Journal of Applied Mechanics, 79(5) (2012).

[11] F.E. Udwadia. Some results on maximum entropy distributions for parameters known to lie in finite intervals. SIAM Review, 31(1):103-109 (1989).

[12] Wolfram Research, Inc., Mathematica, version 12.0, Champaign, IL, USA, 2019. 